\title{
A Normalized Frequency Domain Energy Operator for Broken Rotor Bar Fault Diagnosis
}

\author{
Haiyang Li, Guojin Feng, Member, IEEE, Dong Zhen, Member, IEEE, Fengshou Gu, and Andrew D. Ball
}

\begin{abstract}
In the motor current signal, the characteristic frequency of broken rotor bar (BRB) fault is modulated by the supply frequency and it decreases with the decrease of the load, resulting it to be easily buried under light load conditions. TeagerKaiser energy operator (TKEO) has shown better performance to detect BRB faults than classical methods, such as envelope and spectral analysis. However, the original definition of TKEO leads to its result lack of physical meanings and the causal processing in TKEO can lead to phase distortion and non-ideal filter characteristics. Therefore, this paper proposes a normalized frequency domain energy operator (FDEO) for the BRB fault diagnosis, which does not require causal processing and calculates multiple differentiations in the frequency domain with equal accuracy in one operation. Furthermore, normalized FDEO removes the influence of the supply frequency followed by spectral analysis to extract fault features. The mathematical model of induction motor under healthy and faulty condition are studied in this article. Then, the proposed approach is experimentally validated with seeded one and two BRB faults operating under various load conditions. To verify the effectiveness, the results are compared with TKEO, envelope and spectral analysis. It was found that the proposed method provides slightly obvious fault features with respect to TKEO, especially when the IMs run under light load conditions with two BRB faults.

Index Terms - Broken rotor bar, Induction motor, Motor current signature analysis, Frequency domain energy operator, Fault diagnosis.
\end{abstract}

\section{NOMENCLATURE}

$A(t) \quad$ instantaneous amplitude

$A_{\text {fault }} \quad$ the amplitude of the fault frequency for fault

case

$A_{\text {normal }} \quad$ the amplitude of the fault frequency for

normal case

$\beta \quad$ the modulation index

Envsq $[x(t)] \quad$ the squared envelope of $x(t)$

$\mathcal{F} \quad$ Fourier transform

$\mathcal{F}^{-1} \quad$ inverse Fourier transform

$f_{F} \quad$ BRB fault characteristic frequency

$f_{s} \quad$ supply frequency

$f_{r} \quad$ rotating frequency

$I_{m} \quad$ the amplitude of the supply current
$n_{F}$

$N_{b}$

$p$

$S$

$S_{S}$

$S_{r}$

$\omega$

$\omega_{F}$

$\omega_{s}$

$\omega(t)$

$x(t)$

$\tilde{x}(t)$

$x(n)$

$\psi[x(t)]$

$\overline{\psi[x(t)]}$

signal

$\psi_{N}[x(t)]$

$\psi_{N, \text { main }}\left[i_{F}(t)\right]$ motor stator current

$\varnothing(t)$

instantaneous phase

\section{INTRODUCTION}

$\mathbf{I}^{\mathrm{n}}$ nduction motors (IMs) have been widely used as crucial components in various industrial applications, such as automotive, mining, manufacturing, railway and agriculture, etc. due to its simplicity, reliability and high efficiency [1]. Under tough working conditions, like overrated loads and unexpected events, various types of faults can be induced on the IMs [2-3]. According to the literatures in [3-5], the faults in IMs can be categorized into four types, including bearing fault, stator short winding fault, eccentricity fault and broken rotor bar (BRB) fault. These faults can decrease the productivity, efficiency and reliability of the IMs while increasing the maintenance cost. It is a significant and challenging task to accurately detect early stage faults in IMs, hence effectively

Huddersfield, Huddersfield, HD1 3DH, U.K. (e-mail: Haiyang.Li@hud.ac.uk, G.Feng@hud.ac.uk, F.Gu@hud.ac.uk and A.Ball@hud.ac.uk).

Dong Zhen is with Tianjin Key Laboratory of Power Transmission and Safety Technology for New Energy Vehicles, School of Mechanical Engineering, Hebei University of Technology, Tianjin, 300401, P. R. China. (email: d.zhen@hebut.edu.cn).

the stator current of an IM with BRB fault

The stator current in a healthy IM

the number of rotor bars of the IM

the number of pole pairs of the IM

slip ratio

the rotor rotating speed

angular frequency

angular fault frequency

angular supply frequency

the instantaneous frequency

a continuous signal

the Hilbert transform of $x(t)$

a discrete signal

the instantaneous TKEO of $x(t)$
the mean value of the energy operator of the

a normalized energy operator

main term of the normalized FDEO of the

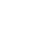

This research was funded in part by the National Natural Science Foundation of China, grant number 51605133, 51705127 and the Hebei Provence Science and Technology Support Program, grant number 17394303D. (Corresponding author: Guojin Feng, Dong Zhen)

Haiyang Li, Guojin Feng, Fengshou Gu and Andrew David Ball are with the Centre for Efficiency and Performance Engineering (CEPE), University of 
avoiding unscheduled downtime, unexpected breakdown and associated operational and maintenance cost.

Many sensing technologies have been developed for the early fault detection of IMs in recent decades, such as acoustic emission analysis [6-7], vibration signature analysis [10-11], acoustic signature analysis [8-9], motor current signature analysis (MCSA) [12-14] and thermal analysis [15-17] etc. These technologies have greatly promoted the development of condition monitoring and fault diagnosis for IMs. In [18], through comparing the detection results based on vibration, motor current and acoustic emission, it was found that the vibration responses are sensitive to bearing faults while MCSA is more sensitive to the rotor faults. Acoustic emission signal, which contains less noises and interference components within the analysing frequency band, is widely used for bearing defects identification [1]. Among all the sensing technologies, motor current from IMs is mostly employed due to that the current sensors are relatively inexpensive and easy to use, and they typically have already been installed in motor drive systems for control purpose in industrial applications [3].

As BRB fault is a major type of rotor failure in IMs, the detection of it based on MCSA in combination with advanced signal processing methods has attracted the attention of researchers and become the research hotspot in developing automated diagnostic processes for IMs. Gu et al. [14] developed an indicator named the modulation signal bispectrum based sideband estimator (MSB-SE) to extract the accurate fault features for BRB fault detection based on MCSA. Naha et al. [19] proposed spectral and subspace decomposition analysis to detect half BRB as well as full BRB faults, especially for the detection of low-amplitude fault sidebands based on MCSA. Abd-el-Malek et al. [20] developed a fast and accurate fault location detection method for BRB fault detection through analysing the stator current envelope using Hilbert transformation. Singh et al. [21] proposed a method to diagnose half BRB fault using motor square current multiple signal classification in a variable frequency drive induction motor. Sang et al. [22] proposed single-phase rotation test which has the advantages that not affected by the asymmetry, load and speed errors. Rotating the rotor manually and reducing supply voltage are required for implementation. Anik et al. [23] delivered Rarleigh quotient technique and extended-Kalman based on MCSA can be used to remove the supply frequency and make the BRB related sideband obvious. Maria et al. [24] presented an intelligent multi-agent system with an overall accuracy ranges from $81.3 \%$ to $89.4 \%$ to diagnose the rotor bar status of IM.

When BRB fault appears in the IMs, no current flows and extra magnetic flux are generated around the fault region and a non-zero backward rotating filed will be generated, resulting a slip frequency with respect to the rotor [25]. This induces fault harmonic components in the stator current which are modulated as sidebands around the supply frequency [26]. Hence, the motor current signal of IMs with BRB faults shows an obvious modulation characteristic, where the BRB fault-related information is the modulated with power supply signal. The main purpose of the signal processing methods is to identify the sidebands of BRB fault frequency around the supply frequency. The popular algorithms include envelope analysis based on Hilbert transform [20], modulation signal bispectrum [14], extend Park's vector approach [27].

In recent years, the TKEO has been investigated and widely used for machinery fault diagnosis, including gearbox [28-29] and bearing [30-31]. Also, it has been investigated for the BRB fault diagnosis as an effective time domain method [32-33]. The TKEO was originally proposed by Teager to observe the flow of oral air during phonation [34], and then further developed by Kaiser et al [35-36] for the amplitude and frequency demodulation in speech analysis. It was well-known as the TKEO with the ability of separating the mono-component signal through calculating its energy as the product of the square of the instantaneous amplitude and frequency of the signal [28]. The TKEO can be directly calculated in the time domain and only three adjacent samples are required for the energy computation, allowing good real-time performance with excellent time resolution. However, such a calculation is a causal processing that can lead to phase distortion and non-ideal filter characteristics [37]. Through analysing the definition of TKEO, it was found that the TKEO is roughly equal to the squared envelope of the derivative of a signal when the modulation component changes slowly [38]. However, the derivative of the original signal is generally different from the signal itself. Therefore, Randall and Smith [37-38] proposed a FDEO which calculates the energy operator by Hilbert transform in the frequency domain and uses non-causal processing. The advantages of the FDEO have been validated in the applications for gear and bearing fault detection where the real-time processing is not critical [37].

This paper investigates the usage of FDEO for the fault diagnosis of IMs with BRB faults. An effective way to remove the effects of the supply frequency on fault feature identification and extraction is proposed based on the normalized FDEO. Then, the proposed approach is validated experimentally on IMs with seeded one BRB and two BRB faults operating under various load conditions. Furthermore, the detection accuracy of FDEO is compared with the TKEO.

The rest of the paper is organized as follows: Section II introduces the mathematical definition of TKEO and FDEO algorithm. Section III presents the motor current signal model with BRB fault, discusses the effects of energy operator on the motor current signal and details the procedure of the proposed method for BRB fault diagnosis of IMs. An experimental test rig is setup in Section IV, followed by the performance evaluation and results discussion in Section V. Finally, the conclusions are drawn in Section VI.

\section{MATHEMATICAl DeFinition OF ENERGy OPERATOR}

For a mono-component signal with slowly changing modulation component, the estimation of its energy operator can be calculated either in the time or frequency domain. This section gives the mathematical definition of the calculations.

\section{A. Teager-Kaiser Energy Operator}

The energy operator was firstly proposed by Teager and 
subsequently improved by Kaiser for speech analysis applications [34-36]. It can be expressed in both continuous and discretized forms [33].

For a continuous signal $x(t)$, a continuous form of the TKEO can be expressed as:

$$
\psi[x(t)]=\dot{x}^{2}(t)-x(t) \cdot \ddot{x}(t)
$$

where $\dot{x}(t)$ and $\ddot{x}(t)$ are the first and second derivative of $x(t)$ which can be presented as $\dot{x}(t)=d x(t) / d t, \ddot{x}(t)=$ $d^{2} x(t) / d t^{2}$ respectively. $\psi[x(t)]$ is the instantaneous energy operator of $x(t)$.

For a discrete signal sampled from a continuous signal with a time interval of $\Delta t$, the discrete time signal can be represented as

$$
x[n]=x(n \Delta t) \text { with } n=0,1,2, \cdots
$$

Then, (1) can be represented in the discrete domain using the approximation of the derivatives backward [32], as shown in (3).

$$
\begin{gathered}
\psi(x[n])=\left(\frac{x[n]-x[n-1]}{\Delta t}\right)^{2}-x[n] \cdot \frac{x[n]-2 \cdot x[n-1]+x[n-2]}{\Delta t^{2}}= \\
\frac{1}{(\Delta t)^{2}}\left(x^{2}[n-1]-x[n-2] \cdot x[n]\right)
\end{gathered}
$$

By scaling and centring, (3) can be written as:

$$
\psi(x[n])=x^{2}[n]-x[n-1] \cdot x[n+1]
$$

From (4), it can be observed that the TKEO of a signal can be efficiently computed from only three adjacent samples in the time domain, which also indicates that the causal processing must be used to retain the advantage for real-time applications [37].

Generally, for a signal $x(t)=A(t) \cos \emptyset(t)$ where $A(t)$ and $\emptyset(t)$ represents the instantaneous amplitude and phase, respectively. The instantaneous frequency $\omega(t)$, can be calculated by $\omega(t)=\dot{\varnothing}(t)$. When the modulation component changes slowly, the $\dot{A}(t)$ and $\dot{\omega}(t)$ are very small and can be ignored [39]. The derivatives of $x(t)$ can be calculated as:

$$
\begin{aligned}
\dot{x}(t) & \approx-\omega(t) A(t) \sin \Phi(t) \\
\ddot{x}(\mathrm{t}) & \approx-\omega^{2}(t) A(t) \cos \Phi(t) \\
\dddot{x}(\mathrm{t}) & \approx \omega^{3}(t) A(t) \sin \Phi(t)
\end{aligned}
$$

Hence, the TKEO of the signal $x(t)$ and $\dot{x}(t)$ can be obtained as,

$$
\begin{gathered}
\psi[x(t)]=[\dot{x}(t)]^{2}-x(t) \ddot{x}(t) \approx \omega^{2}(t) A^{2}(t) \\
\psi[\dot{x}(t)]=[\ddot{x}(t)]^{2}-\dot{x}(t) \dddot{x}(t) \approx \omega^{4}(t) A^{2}(t)
\end{gathered}
$$

By combining (8) and (9), the instantaneous amplitude $A(t)$ and instantaneous frequency $\omega(t)$ can be achieved by:

$$
\left\{\begin{array}{l}
A(t)=\frac{\psi[x(t)]}{\sqrt{\psi[\dot{x}(t)]}} \\
\omega(t)=\sqrt{\frac{\psi[\dot{x}(t)]}{\psi[x(t)]}}
\end{array}\right.
$$

\section{B. Frequency Domain Energy Operator}

Instead of being calculated in the time domain, the energy operator can also be obtained in the frequency domain, in which case it was named as FDEO. According to (5) [37], the energy operator of the signal $x(t)$ in (8) is actually approximated to the squared envelope of $\dot{x}(t)$, which is the sum of the squares of $\dot{x}(t)$ and its Hilbert transform. Suppose Envsq $[x(t)]$ represents the squared envelope of $x(t)$, it can be expressed as:

$$
\operatorname{Envsq}[x(t)]=x^{2}(t)+\tilde{x}^{2}(t)
$$

where, $\tilde{x}(t)$ is the Hilbert transform of $x(t)$ in the frequency domain. By combining (5), the envelope square of $\dot{x}(t)$ can be obtained as:

$$
\operatorname{Envsq}[\dot{x}(t)] \approx[-\omega(t) A(t)]^{2}=\omega^{2}(t) A^{2}(t)
$$

Furthermore, based on the derivative property of Fourier transform, $\dot{x}(t)$ can be achieved in the frequency domain by inversing Fourier transformation of the product of $j \omega$ and can be expressed as follows:

$$
\dot{x}(t)=\mathcal{F}^{-1}[j \omega \mathcal{F}(x(t))]
$$

where $\omega$ represents angular frequency and $j$ represents the imaginary axis. $\mathcal{F}^{-1}$ and $\mathcal{F}$ represent inverse Fourier transform and Fourier transform respectively.

From (8), the TKEO can be expressed as:

$$
\psi[x(t)] \approx \operatorname{Envsq}[\dot{x}(t)]
$$

Since,

$$
A^{2}(t)=\operatorname{Envsq}[x(t)]
$$

by combining (8), the square of the instantaneous frequency can be written as:

$$
\omega^{2}(t)=\operatorname{Envsq}[\dot{x}(t)] / \operatorname{Envsq}[x(t)]
$$

As both derivate of the signal and its envelope are obtained in the frequency domain. Therefore, the FDEO of a signal $x(t)$ can be calculated using (14) and the instantaneous amplitude and instantaneous frequency can be obtained by (17) in the frequency domain.

$$
\left\{\begin{array}{l}
A(t)=\sqrt{\operatorname{Envsq}[x(t)]} \\
\omega(t)=\sqrt{\operatorname{Envsq}[\dot{x}(t)] / \operatorname{Envsq}[x(t)]}
\end{array}\right.
$$

\section{APPLICATION OF ENERGY OPERATOR ON BRB FAULT DIAGNOSIS}

\section{A. Mathematical Model of Induction Motor Current}

The BRB fault of IMs produces periodic disturbances and a modulation component will be induced in the motor current [40-41]. The principal BRB fault characteristic frequency $f_{F}$, can be represented by:

$$
f_{F}=2 s f_{s}
$$

where $s$ and $f_{s}$ are the slip ratio and supply frequency, respectively. The motor slip ratio $s$ is related to the motor operating speed as shown in (19).

$$
s=\frac{s_{s}-S_{r}}{S_{S}}=\frac{f_{S} / p-f_{r}}{f_{s} / p}
$$

where $S_{s}$ is the synchronize speed, $S_{r}$ denotes the rotor speed, $f_{r}$ represents rotating frequency and $p$ represents the number of pole pairs of the IM.

The stator current in a healthy $\mathrm{IM}, i_{N}(t)$, is purely sinusoidal 
when the IM is powered by a sinusoidal voltage system with the main supply frequency of $f_{s}(50 \mathrm{~Hz} / 60 \mathrm{~Hz})$, it can be presented by:

$$
i_{N}(t)=I_{m} \cos \left(w_{s} \mathrm{t}\right)=I_{m} \cos \left(2 \pi f_{s} \mathrm{t}\right)
$$

where $\omega_{s}=2 \pi f_{s}, I_{m}$ represents the amplitude of the supply current. In an IM with BRB faults, periodical perturbations will be caused by the fault, leading to the stator current being amplitude modulated by the fault frequency $f_{F}$ in terms of amplitude modulation [33]. Therefore, the stator current of an IM with BRB fault, $i_{N}(t)$, can be expressed by:

$$
\begin{aligned}
i_{F}(t) & =i_{N}(\mathrm{t}) \cdot\left[1+\beta \cos \left(\omega_{F} t\right)\right] \\
& =I_{m} \cos \left(2 \pi f_{s} t\right)+\frac{I_{m} \beta}{2} \cos \left[2 \pi\left(f_{s}+f_{F}\right) t\right] \\
& +\frac{I_{m} \beta}{2} \cos \left[2 \pi\left(f_{s}-f_{F}\right) t\right]
\end{aligned}
$$

where $\omega_{F}=2 \pi f_{F}$, and $\beta$ denotes the modulation index related to the fault severity of BRB and can be calculated using (22) [31].

$$
\beta \approx n_{F} / N_{b}
$$

where $n_{F}$ denotes the number of BRBs while $N_{b}$ denotes the number of rotor bars of the IM.

Generally, $\beta \ll 1$ due to the $n_{F} \ll N_{b}$, especially for the early stage fault of BRB. Therefore, the amplitudes of the faultrelated sideband frequency, $f_{S} \pm f_{F}$, are much smaller compared to the amplitude of the supply frequency $f_{s}$.

\section{B. Analysis of Energy Operator on the Motor Current Signal}

Apply the energy operator on the motor current signal under normal condition as presented in the (20), we can get:

$$
\psi\left[i_{N}(t)\right]=I_{m}^{2} \omega_{s}^{2}
$$

It shows that the energy operator of the motor current in normal condition is a constant value corresponding to the squared envelope of the motor current signal. Then apply the energy operator to the motor current from a faulty IM demonstrated by (21), and ignore the terms that are multiplied by $\beta^{2}$ due to $\beta \ll 1$, it is given as follows:

$$
\begin{array}{r}
\psi\left[i_{F}(t)\right]=I_{m}^{2} \omega_{s}^{2}+\frac{\beta}{2} I_{m}^{2}\left(4 \omega_{s}^{2}+\omega_{F}^{2}\right) \cos \left(\omega_{F} t\right)+ \\
\frac{\beta}{2} I_{m}^{2} \omega_{F}^{2}\left\{\cos \left[\left(2 \omega_{s}+\omega_{F}\right) t\right]+\cos \left[\left(2 \omega_{s}-\omega_{F}\right) t\right]\right\}
\end{array}
$$

As shown in (24), there are mainly four terms in the energy operator of the faulty motor current signal, including a constant term related to the supply frequency, a fluctuating term related to the fault frequency and two fluctuating sideband terms around twice of the supply frequency. Obviously, the fluctuating term that related to the fault frequency $\omega_{F}$ is more significant and can be extracted for the fault detection with higher accuracy. To highlight the fluctuating term related to the fault frequency and remove the constant term, a normalized energy operator, $\psi_{N}[x(t)]$, can be calculated based on the FDEO of the motor current signal and can be given as follows [33]:

$$
\psi_{N}[x(t)]=\frac{\psi[x(t)]-\overline{\psi[x(t)]}}{\overline{\psi[x(t)]}}
$$

where $\overline{\psi[x(t)]}$ is the mean value of the energy operator of the signal $x(t)$.

Hence, the normalized FDEO of the motor stator current for a normal IM in (23) and faulty IM in (24) can be represented as follows:

$$
\begin{gathered}
\psi_{N}\left[i_{N}(t)\right]=0 \\
\psi_{N}\left[i_{F}(t)\right]=\frac{\beta}{2}\left[4+\left(\frac{\omega_{F}}{\omega_{S}}\right)^{2}\right] \cos \left(\omega_{F} \mathrm{t}\right)+ \\
\frac{\beta}{2}\left(\frac{\omega_{F}}{\omega_{s}}\right)^{2}\left\{\cos \left[\left(2 \omega_{s}+\omega_{F}\right) t\right]+\cos \left[\left(2 \omega_{s}-\omega_{F}\right) t\right]\right\}
\end{gathered}
$$

As shown in (27), the supply frequency and the DC components are removed, and the main term of the normalized FDEO of the motor stator current that related to the fault frequency $\omega_{F}$, as shown in (28), can be separated and employed to indicate the fault type and severity.

$$
\psi_{N, \text { main }}\left[i_{F}(t)\right]=\frac{\beta}{2}\left[4+\left(\frac{\omega_{F}}{\omega_{s}}\right)^{2}\right] \cos \left(\omega_{F} t\right)
$$

\section{Proposed Method for BRB Fault Diagnosis}

Based on the mathematical model of the energy operator and motor current signal, a method for the BRB fault diagnosis is proposed, which has meaningful physical explanations using FDEO. The main steps of the proposed method are listed as follows and presented in Fig. 1.

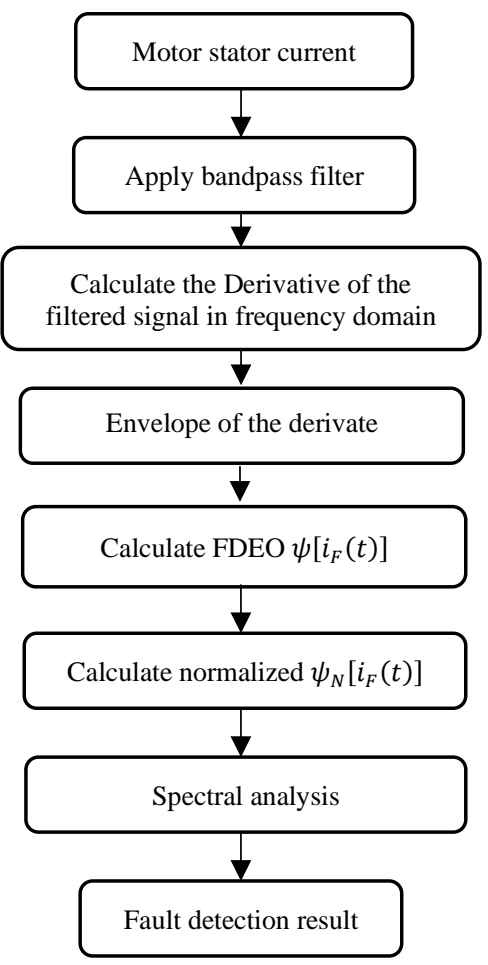

Fig. 1. Flow chart of the proposed method for BRB fault diagnosis

(1) Apply a band-pass filter to pre-process the measured motor current signal. As the motor slip is normally less than 7\% [42], the BRB fault frequencies modulated on the supply frequency are usually less than $7 \mathrm{~Hz}$ when the supply frequency is $50 \mathrm{~Hz}$. Therefore, the pass band for the filter in this research work is set from $35 \mathrm{~Hz}$ to $65 \mathrm{~Hz}$;

(2) Calculate zero phase shift derivative of the filtered motor current signal.

(3) Calculate FDEO $\psi\left[i_{F}(t)\right]$ of the motor current signal using (12) in combination with envelope analysis based on Hilbert transform, and then calculate the normalized energy 
operator $\psi_{N}\left[i_{F}(t)\right]$ by using (25);

(4) Employ the spectral analysis to reveal the frequency characteristics of the normalized energy operator;

(5) Evaluate the main term of the normalized energy operator $\psi_{N}\left[i_{F}(t)\right]$ which is related to the fault frequency $\omega_{F}$ presented in (28). Look for the frequency components corresponding to the BRB fault characteristic frequency according to (18) and extract its amplitude to indicate the fault severities of BRB fault.

\section{EXPERIMENTAL SETUP}

To validate the performance of the proposed method for BRB fault diagnosis, an IM test rig is set up as shown in Fig. 2. Three industrial motors with the same specification are tested with sensorless control mode and their specifications are listed in Table I. Among them, one of the motors is kept as healthy regarding to the normal case, and other two motors are seeded with one and two BRB fault regarding to the cases of $1 \mathrm{BRB}$ and $2 \mathrm{BRB}$, respectively. The BRB faults were artificially produced by drilling one bar and two bars to its full depth for seeding 1 BRB and 2 BRB faults, as shown in Fig. 3.

TABLE I

SPECIFICATION OF TEST MOTORS

\begin{tabular}{lll}
\hline \multicolumn{1}{c}{ Parameters } & & Value \\
\hline Rated voltage $(\Delta / \mathrm{Y})$ & $230 / 400$ \\
Rated current $(\Delta / \mathrm{Y})$ & $15.9 / 9.2 \mathrm{~A}$ \\
Motor power & $4 \mathrm{~kW}$ \\
Number of phases & 3 \\
Number of poles & 4 Poles \\
Supply frequency & $50 \mathrm{~Hz}$ \\
Rated speed & 1420 \\
Number of rotor bars & 28 \\
\hline \hline
\end{tabular}
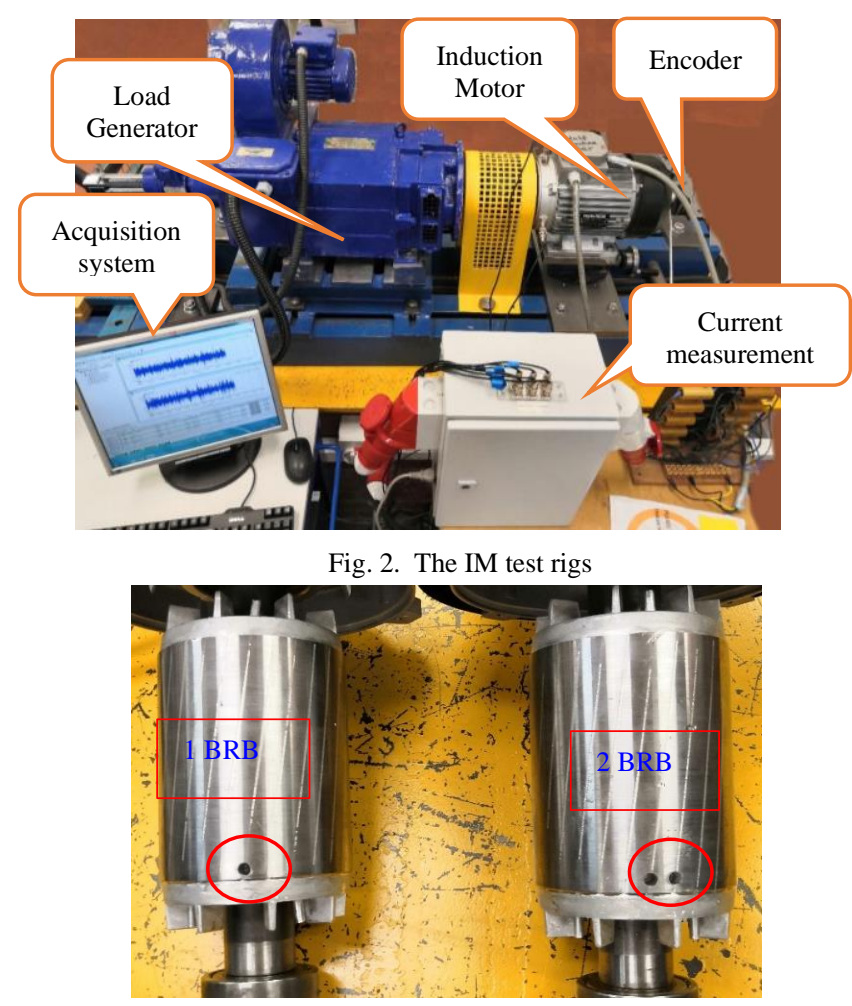

Fig. 3. The simulated BRB faults

For the three cases, the tests were carried out at the rated speed under different operating loads. The motor is mechanically coupled to a generator to adjust load conditions. Table II summarizes the operating condition of the experimental tests for the three cases, where the loads were set to five conditions, ranging from $0 \%$ to $80 \%$ of the rated load with an interval of $20 \%$. The hall-effect current sensors are used to measure the motor stator current. In every case, 30 seconds of three phase current signals were recorded at the sampling frequency of 96 $\mathrm{kHz}$ through a data acquisition system.

TABLE II

TEST OPERATING CONDITIONS

\begin{tabular}{ccccccccc}
\hline \hline $\begin{array}{c}\text { Fault } \\
\text { Case }\end{array}$ & $\begin{array}{c}\text { Expected } \\
\text { Speed } \\
(\%)\end{array}$ & & $\begin{array}{c}\text { Load } \\
(\%)\end{array}$ & & $\begin{array}{c}\text { Sampling } \\
\text { Frequency } \\
(\mathrm{kHz})\end{array}$ & $\begin{array}{c}\text { Data } \\
\text { Length } \\
(\mathrm{s})\end{array}$ \\
\hline Normal & 100 & 0 & 20 & 40 & 60 & 80 & 96 & 30 \\
1BRB & 100 & 0 & 20 & 40 & 60 & 80 & 96 & 30 \\
2BRB & 100 & 0 & 20 & 40 & 60 & 80 & 96 & 30 \\
\hline \hline
\end{tabular}

\section{Performance Evaluation and Results Discussion}

\section{A. Experimental Data Analysis in the Time and Frequency Domain}

Recall the theoretical discussion in Section III, the periodic disturbances produced by BRB faults are modulated on the supply frequency component, i.e. 50Hz. Fig. 4 shows the waveform and spectrum of the measured motor current signal for the case of normal and 2 BRB under $80 \%$ of rated load condition.
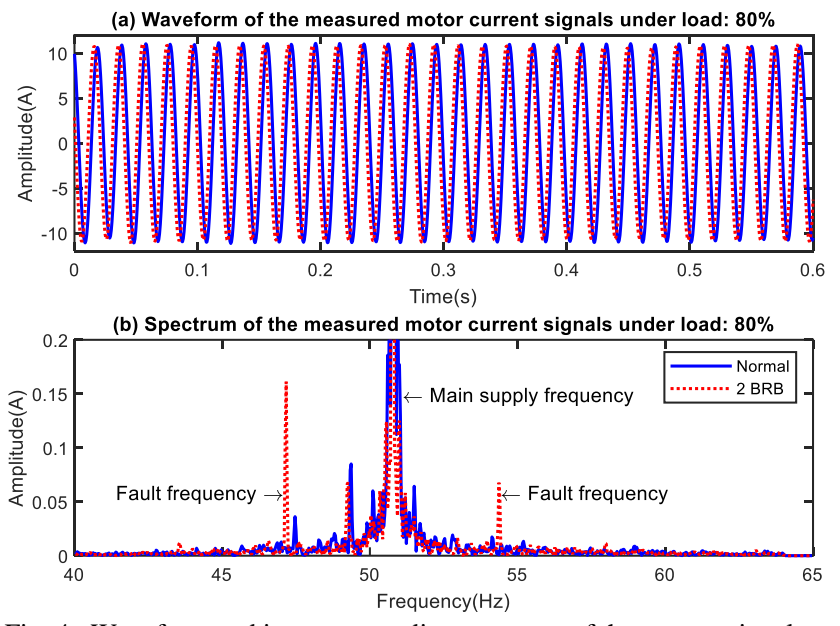

Fig. 4. Waveform and its corresponding spectrum of the current signals From the time domain signal in Fig. 4 (a), it's difficult to observe the modulation effect and the dissimilarities of the motor current signals under the normal and faulty conditions. However, the fault characteristics can be clearly identified in the frequency domain as shown in Fig. 4 (b) and can be easily extracted for the BRB fault detection. Recall the calculation in (18), the BRB fault characteristic frequency is determined by the motor slip ratio $s$ and the supply frequency $f_{s}$. The supply frequency $f_{s}$ is close to the electrical power supply frequency, i.e. $50 \mathrm{~Hz}$. With the sensorless control mode, the motor control system is a feedback one which can adjust the supply frequency to achieve the expected rotating speed. That is the reason why the supply frequency changes slightly with the operating condition of the IM. Table III shows the estimated supply frequency for the three cases under different operating 
conditions based on the spectral analysis. It can be observed that the estimated supply frequency slightly increases with the increment of loads, and slightly decreases with the increase of the fault severity.

TABLE III

MAIN SUPPLY FREQUENCY UNDER DIFFERENT OPERATING CONDITIONS

\begin{tabular}{lcrcccc}
\hline \hline & Speed & \multicolumn{5}{c}{ Main supply frequency $\boldsymbol{f}_{\boldsymbol{s}}(\mathrm{Hz})$} \\
\cline { 3 - 7 } Case & $(\%)$ & Load & Load & Load & Load & Load \\
& & $0 \%$ & $20 \%$ & $40 \%$ & $60 \%$ & $80 \%$ \\
\hline Normal & 100 & 49.40 & 49.74 & 50.10 & 50.46 & 50.80 \\
1BRB & 100 & 49.40 & 49.73 & 50.10 & 50.45 & 50.79 \\
2BRB & 100 & 49.39 & 49.72 & 50.07 & 50.43 & 50.77 \\
\hline \hline
\end{tabular}

TABLE IV

ROTOR SPEED IN HZ UNDER DIFFERENT OPERATING CONDITIONS

\begin{tabular}{lcrrrrr}
\hline \hline \multirow{3}{*}{ Case } & Speed & \multicolumn{5}{c}{ Rotor speed in $\mathrm{Hz} \boldsymbol{f}_{\boldsymbol{r}}(\mathrm{Hz})$} \\
\cline { 3 - 7 } & & Load & Load & Load & Load & Load \\
& & $0 \%$ & $20 \%$ & $40 \%$ & $60 \%$ & $80 \%$ \\
\hline Normal & 100 & 24.65 & 24.65 & 24.64 & 24.63 & 24.57 \\
1BRB & 100 & 24.65 & 24.64 & 24.64 & 24.62 & 24.55 \\
2BRB & 100 & 24.64 & 24.63 & 24.61 & 24.58 & 24.48 \\
\hline \hline
\end{tabular}

Recall the definition of motor slip ratio in (19), it is related to the main supply frequency $f_{s}$, the rotor speed $f_{r}$ and the number of pole pairs $p$ ( $p=2$ in this work). The rotor speed for the three cases under different operating loads were calculated and shown in Table IV. Then, the motor slip ratio is obtained by (19) and presented in Table V. It can be seen that the rotor speed slightly decreases when the operating load or fault severity increases, while the motor slip ratio increases with the increasing of the operating loads and fault severities. Thereafter, the fault characteristic frequency of the three cases under different operating conditions are calculated and shown in Table VI, it can be observed that the amplitudes of the fault characteristic frequency are very low under light load conditions (0\% and 20\% loads), and they can be easily submerged by the spectral leakage of the supply frequency.

$$
\text { TABLE } \mathrm{V}
$$

MOTOR SLIP RATIO UNDER DIFFERENT OPERATING CONDITIONS

\begin{tabular}{lcccccc}
\hline \hline & Speed & \multicolumn{5}{c}{ Slip ratio s (\%) } \\
\cline { 3 - 7 } Case & $(\%)$ & $\begin{array}{c}\text { Load } \\
0 \%\end{array}$ & $\begin{array}{c}\text { Load } \\
20 \%\end{array}$ & $\begin{array}{c}\text { Load } \\
40 \%\end{array}$ & $\begin{array}{c}\text { Load } \\
60 \%\end{array}$ & $\begin{array}{c}\text { Load } \\
80 \%\end{array}$ \\
\hline Normal & 100 & 0.19 & 0.89 & 1.63 & 2.38 & 3.28 \\
1BRB & 100 & 0.21 & 0.89 & 1.64 & 2.39 & 3.35 \\
2BRB & 100 & 0.20 & 0.94 & 1.72 & 2.51 & 3.55 \\
\hline \hline
\end{tabular}

TABLE VI

FAULT CHARACTERISTIC FREQUENCY UNDER DIFFERENT OPERATING

\begin{tabular}{lcccccc}
\multicolumn{8}{c}{ CONDITIONS } \\
\hline \hline \multirow{2}{*}{ Case } & Speed & \multicolumn{6}{c}{ Fault characteristic frequency $2 \boldsymbol{2 s f _ { s }}(\mathrm{Hz})$} \\
\cline { 3 - 7 } & & Load & Load & Load & Load & Load \\
& & $0 \%$ & $20 \%$ & $40 \%$ & $60 \%$ & $80 \%$ \\
\hline Normal & 100 & 0.19 & 0.89 & 1.63 & 2.38 & 3.28 \\
1BRB & 100 & 0.21 & 0.89 & 1.64 & 2.39 & 3.35 \\
2BRB & 100 & 0.20 & 0.94 & 1.72 & 2.51 & 3.55 \\
\hline \hline
\end{tabular}

Fig. 5 shows the spectrum of the current signal for the case of 2 BRB fault under different load conditions. Note that it is difficult to identify the modulation sideband caused by the BRB fault under light load conditions, i.e. $0 \%, 20 \%$ and $40 \%$ of the rated load. To evaluate the performance of the fault diagnosis based on spectral analysis, the amplitudes of the modulation sidebands of the spectrums are extracted and used to indicate the fault severities, as shown in Fig. 6 (a).

Fig. 6(a) shows the sideband amplitude change of the fault characteristic frequency according to the load conditions and fault severities. Notice that the fault severities for the cases of normal, 1 BRB and 2 BRB cannot be distinguished under most operating conditions, including $40 \%$ and $60 \%$ of the rated load. For the high load condition, i.e. $80 \%$ of the rated load, the fault severity can be clearly indicated due to the good results obtained in Fig. 5(e). Although the fault severity can be distinguished in Fig. 6(a) under no load condition, i.e. $0 \%$ of the rated load, it cannot be well trusted as a reliable result. This is due to that the amplitudes of the fault characteristic frequency under low operating loads are prone to be submerged by the spectral leakage of the supply frequency, which thereafter will significantly affect the accuracy of the detection results.

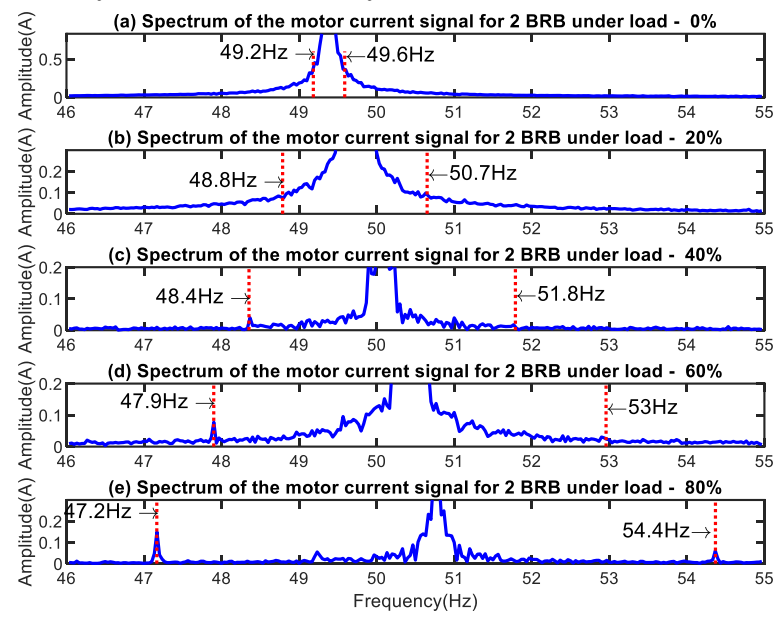

Fig. 5. Spectrum of the current signal under different loads for 2 BRB fault

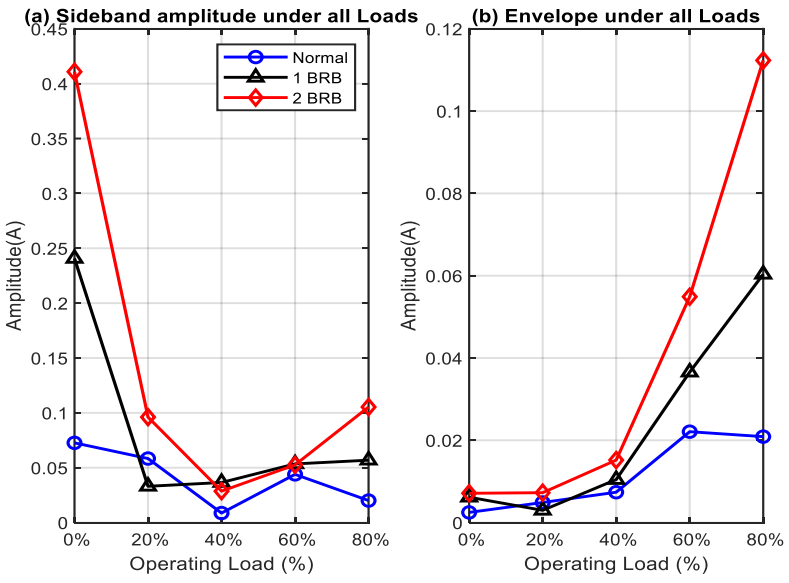

Fig. 6. (a) Spectrum sideband and (b) envelope analysis based BRB fault detection

\section{B. Envelope analysis results and discussion}

Envelope analysis is an effective method for demodulation, which can remove the main carrier components from a modulation signal. It has been widely used in practice for fault diagnosis purposes. Hence, the envelope analysis is also employed to suppress the main supply frequency component of the measured motor current. The envelope spectrum of the measured motor current signals for BRB faults under different operating loads were obtained and presented in Fig. 7. The 
modulation components of the motor current signal related to the fault characteristic frequency are illustrated as $f_{1 B R B}$ and $f_{2 B R B}$ in Fig. 7. The amplitudes of the fault characteristic frequency under high operating loads, such as $40 \%, 60 \%$ and $80 \%$ of the rated load as shown in Fig. 7(c), (d) and (e), are obvious. Similar as those in Fig. 6 (b), the amplitudes of the fault characteristic frequency can be extracted and used as the fault features to represent the severity of the BRB fault under high operating loads, such as $40 \%, 60 \%$ and $80 \%$ of the rated load. However, the fault features under light load conditions, such as $0 \%$ and $20 \%$ of the rated load, are not recognized properly due to the strong noise pollution and interference frequency components.
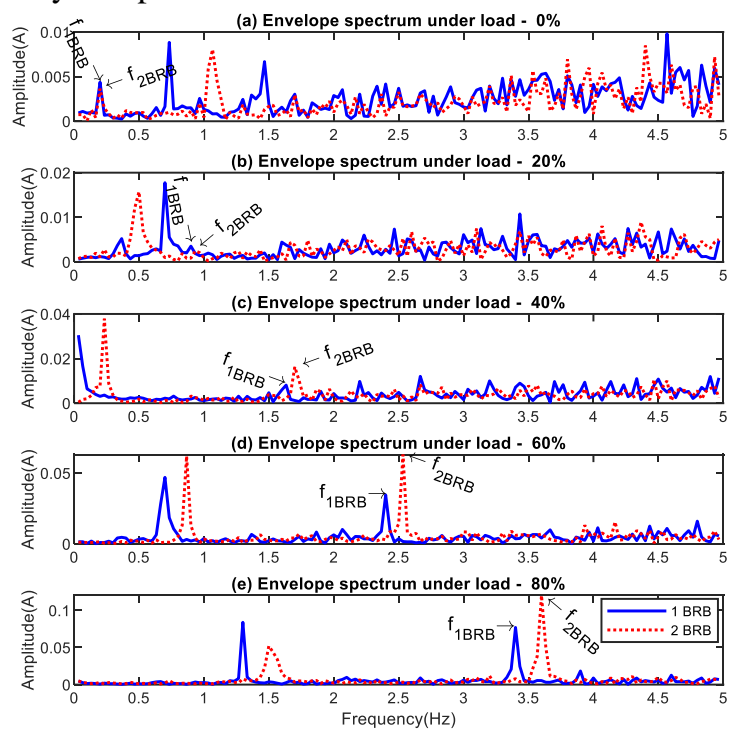

Fig. 7. Envelope spectrum under different loads

\section{Energy Operator Analysis results and discussion}

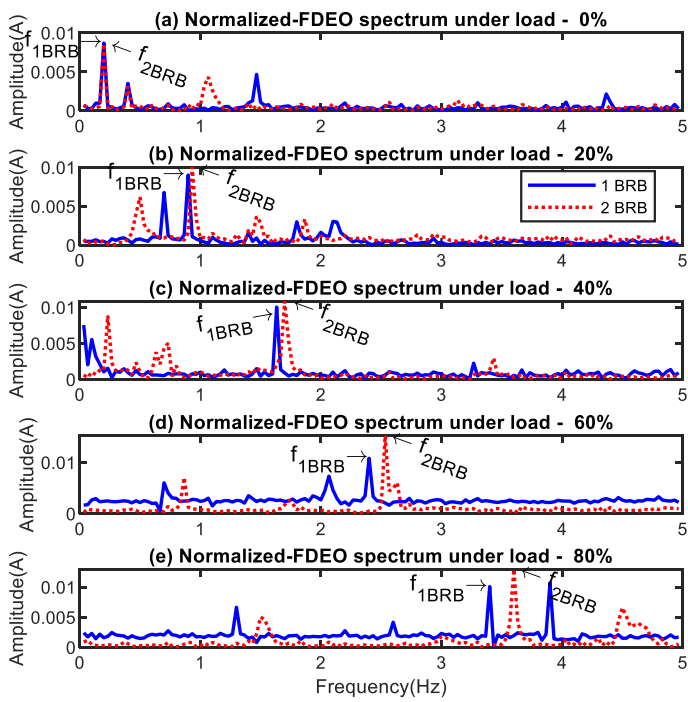

Fig. 8. Normalized-FDEO spectrum under different loads

After the above spectral and envelope analysis, the normalized FDEO by (25) is employed to analyse the measured motor current signals and the normalized FDEO spectrums under different load conditions are shown in Fig. 8. According to (22), the $\beta$ increases with the fault severity. And according to (18)(19), the slip ratio increases with the loads which results in $\omega_{F}$ increases with loads. Therefore, based on (28), the amplitude of the FEDO of the current signal from a faulty IM also increases along the fault severity and loads. As the analysis results shown in Fig. 8, the BRB fault characteristic frequency can be clearly observed under all load conditions and they can be easily extracted with no serious interference components. Moreover, the amplitudes of the fault characteristic frequencies increase when the operating loads increase, and the differentiation is more noticeable for most of the load conditions. Such experimental data analysis result also shows good consistency with theoretical analysis.
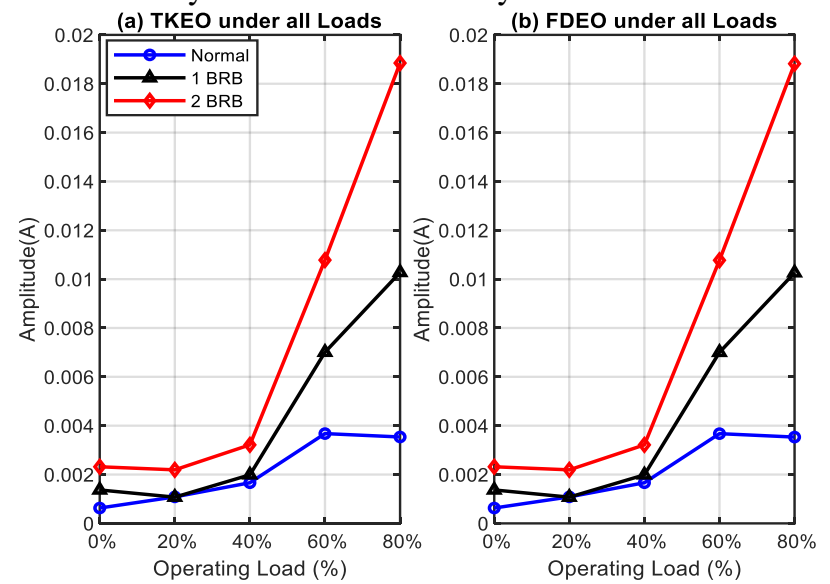

Fig. 9. The amplitude at BRB fault frequency using (a) normalized TKEO and (b) normalized FDEO under all operating conditions

Similarly, the normalized TKEO spectrum can be calculated. By extracting the amplitudes of the BRB fault characteristic frequency under different load conditions, the results for the normalized TKEO and FDEO are presented in Fig. 9 (a) and (b), respectively. As can be observed, these two methods show similar detection results, which outperform that of the envelope-based analysis result in Fig. 6(b), especially for the fault cases under light load conditions, such as $0 \%$ and $20 \%$ of the rated load.

(a) RD Comparisons via methods for 1 BRB diagnosis

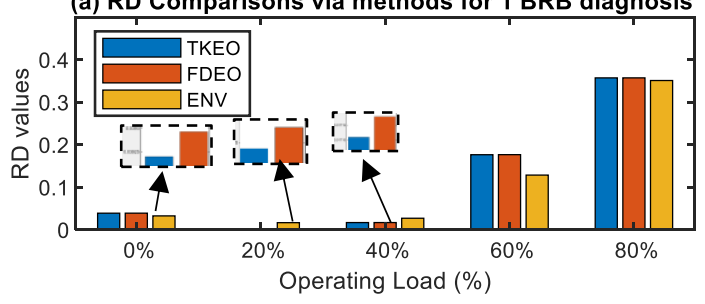

(b) RD Comparisons via methods for 2 BRB diagnosis

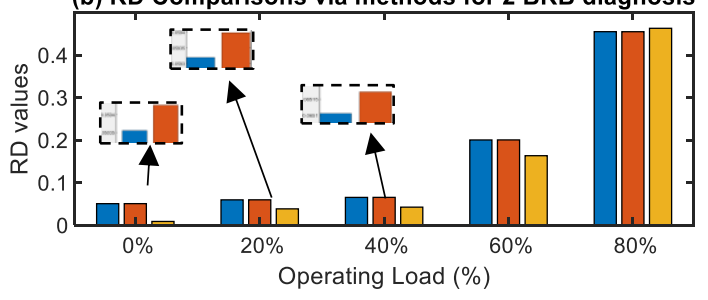

Fig. 10. Comparisons of the RD of the detection results based on the three methods

Furthermore, the performance and precisions of the three methods, including TKEO, FDEO and envelope analysis, are evaluated by calculating the relative differentiation (RD) of the detection results using (29) and the results are presented in Fig. 10. 


$$
R D=\frac{A_{\text {fault }}-A_{\text {normal }}}{M a x\left(A_{\text {fault and normal }}\right)}
$$

where $A_{\text {fault }}$ is the fault feature value (the amplitude of the fault characteristic frequency) of the cases of $1 \mathrm{BRB}$ and $2 \mathrm{BRB}$, and $A_{\text {normal }}$ is the feature value of the normal case.

According to the comparison analysis in Fig. 10, it is found that the RD values of the two energy operator based methods for fault detection are higher than that of envelope analysis in most of the fault and operating cases, especially for the fault cases under light load conditions (no load). And the result is more distinct and consistent for the serious faults. Moreover, through analysing the RD values obtained by the two energy operator methods, it demonstrates that they provide similar detection results with higher accuracy compared with envelopebased analysis. In addition, FDEO also shows slightly better results than TKEO which have been partly magnified in Fig.10, especially for the light load conditions, such $0 \%, 20 \%$ and $40 \%$ of the rated load.

Furthermore, the RD of instantaneous amplitude for different fault severities under various load conditions are also compared and shown in Fig. 11, it is found that the RD of instantaneous amplitude by FDEO, TKEO and envelope analysis can provide similar fault feature under most operations. But FDEO and envelope show slightly better fault features than the TKEO for the case of 2 BRB under $0 \%$ and $20 \%$ rated load as shown in Fig. 11 (b). Combining the results in Fig 10 and Fig 11, all the three methods perform well for both 1BRB and 2BRB fault detection under high loads (such as 60\% and 80\% loads). However, FDEO shows better result than the other two for BRB fault detection under light load conditions, especially for no load $(0 \%)$ and $20 \%$ of the rated load.

(a) RD of IA Comparisons via methods for 1 BRB diagnosis
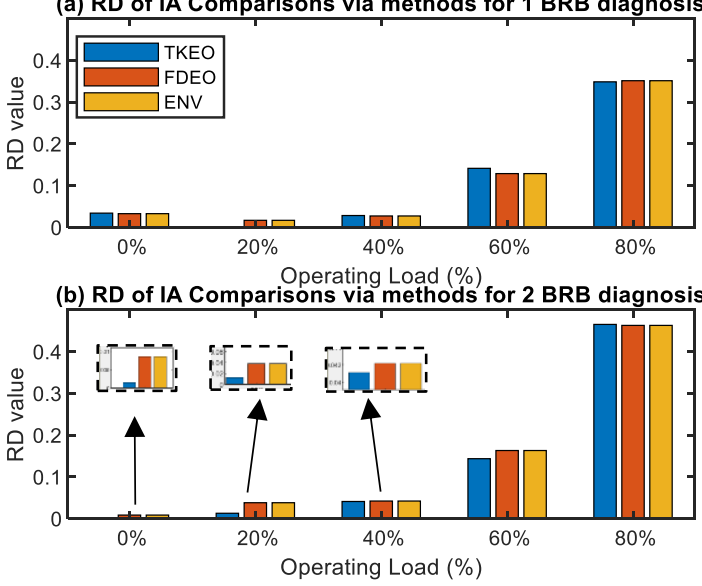

Fig. 11. RD of instantaneous amplitude (IA) comparisons of the detection results based on the three methods

Furthermore, in comparison with other state-of-the-art paper for BRB fault diagnosis, the proposed method uses less computational resources than those in [14][23] and is easier to capture the current signal than the method in [22]. More importantly, the proposed method has solid physical meanings that guarantees its diagnosis accuracy.

\section{CONCLUSION}

In this work, a BRB fault diagnosis method is proposed based on the normalized FDEO of the motor current combined with the spectral analysis. Its performance and efficiency are experimentally validated on IMs for BRB fault diagnosis and compared with the conventional envelope analysis methods. The proposed method obtains diagnosis results with higher distinguishability for BRB fault severities under different operating conditions, especially for the light load conditions. Compared to the TKEO, the proposed method has better physical meanings with non-causal processing and can provide slightly better fault features for BRB fault diagnosis under light load conditions. In addition, the energy operator-based methods can produce more accurate demodulation and better detection results than the conventional envelope analysis. Therefore, the proposed method based on FDEO analysis has the potential to improve the accuracy of fault detection in machinery diagnostics. However, advanced methods need to be investigated to further improve the proposed method for BRB fault diagnosis in future research work.

\section{REFERENCES}

[1]A. Choudhary, D. Goyal, S. L. Shimi, and A. Akula, "Condition Monitoring and Fault Diagnosis of Induction Motors: A Review", Arch. Comput. Methods Eng., vol. 26, no. 4, pp. 1221-1238, Sep. 2019.

[2]Z. Wang, J. Yang, H. Li, D. Zhen, Y. Xu, and F. Gu, "Fault Identification of Broken Rotor Bars in Induction Motors Using an Improved Cyclic Modulation Spectral Analysis", Energies, vol. 12, no. 17, p. 3279, Jan. 2019.

[3] Y. Liu and A. M. Bazzi, "A review and comparison of fault detection and diagnosis methods for squirrel-cage induction motors: State of the art", ISA Trans., vol. 70, pp. 400-409, Sep. 2017.

[4]H. Henao et al., "Trends in Fault Diagnosis for Electrical Machines", in IEEE Industrial Electronics Magazine, 2014, vol. 8, pp. 31-42.

[5]M. Riera-Guasp, J. A. Antonino-Daviu, and G.-A. Capolino, "Advances in Electrical Machine, Power Electronic, and Drive Condition Monitoring and Fault Detection: State of the Art", IEEE Trans. Ind. Electron., vol. 62, no. 3, pp. 1746-1759, Mar. 2015.

[6]Dr. M. R. Islam, J. Uddin, and J. Kim, “Acoustic Emission Sensor Network Based Fault Diagnosis of Induction Motors Using a Gabor Filter and Multiclass Support Vector Machines", Ad Hoc Sens. Wirel. Netw., vol. 34, pp. 273-287, Nov. 2016.

[7]A. Widodo, B.-S. Yang, E. Y. Kim, A. C. C. Tan, and J. Mathew, "Fault diagnosis of low speed bearing based on acoustic emission signal and multiclass relevance vector machine", Nondestruct. Test. Eval., vol. 24, no. 4, pp. 313-328, Dec. 2009.

[8] A. Glowacz, W. Glowacz, Z. Glowacz, and J. Kozik, "Early fault diagnosis of bearing and stator faults of the single-phase induction motor using acoustic signals", Measurement, vol. 113, pp. 1-9, Jan. 2018.

[9]A. Glowacz, "Diagnostics of Rotor Damages of Three-Phase Induction Motors Using Acoustic Signals and SMOFS-20-EXPANDED", Arch. Acoust., vol. 41, no. 3, pp. 507-515, Apr. 2016.

[10] A. Prudhom, J. Antonino-Daviu, H. Razik, and V. Climente-Alarcon, "Time-frequency vibration analysis for the detection of motor damages caused by bearing currents", Mech. Syst. Signal Process., vol. 84, pp. 747-762, Feb. 2017.

[11] D. Zhen, Z. Wang, H. Li, H. Zhang, J. Yang, and F. Gu, "An Improved Cyclic Modulation Spectral Analysis Based on the CWT and Its Application on Broken Rotor Bar Fault Diagnosis for Induction Motors", Appl. Sci., vol. 9, no. 18, p. 3902, Jan. 2019.

[12] D. Z. Li, W. Wang, and F. Ismail, "An Enhanced Bispectrum Technique Monitoring”, IEEE Trans. Instrum. Meas., vol. 64, no. 10, pp. 2679-2687, Oct. 2015.

[13] S. Singh and N. Kumar, "Detection of Bearing Faults in Mechanical Systems Using Stator Current Monitoring", IEEE Trans. Ind. Inform., vol. 13, no. 3, pp. 1341-1349, Jun. 2017.

[14] F. Gu, T. Wang, A. Alwodai, X. Tian, Y. Shao, and A. D. Ball, “A new method of accurate broken rotor bar diagnosis based on modulation signal bispectrum analysis of motor current signals", Mech. Syst. Signal Process., vol. 50-51, pp. 400-413, Jan. 2015.

[15] G. Singh, T. Ch. Anil Kumar, and V. N. A. Naikan, "Induction motor inter turn fault detection using infrared thermographic analysis", Infrared Phys. Technol., vol. 77, pp. 277-282, Jul. 2016. 
[16] Y. Xie, J. Guo, P. Chen, and Z. Li, "Coupled Fluid-Thermal Analysis for Induction Motors with Broken Bars Operating under the Rated Load", Energies, vol. 11, no. 8, p. 2024, Aug. 2018.

[17] D. López-Pérez and J. Antonino-Daviu, "Application of Infrared Thermography to Failure Detection in Industrial Induction Motors: Case Stories", IEEE Trans. Ind. Appl., vol. 53, no. 3, pp. 1901-1908, May 2017.

[18] W. Li and C.K. Mechefske, 'Detection of induction motor faults: a comparison of stator current, vibration and acoustic methods", Journal of vibration and Control, Vol. 12, no. 2, pp.165-188. Feb 2006

[19] A. Naha, A. K. Samanta, A. Routray, and A. K. Deb, "A Method for Detecting Half-Broken Rotor Bar in Lightly Loaded Induction Motors Using Current", IEEE Trans. Instrum. Meas., vol. 65, no. 7, pp. 1614-1625, Jul. 2016. [20] M. Abd-el-Malek, A. K. Abdelsalam, and O. E. Hassan, "Induction motor broken rotor bar fault location detection through envelope analysis of start-up current using Hilbert transform", Mech. Syst. Signal Process., vol. 93, pp. 332-350, Sep. 2017.

[21] G. Singh and V. N. A. Naikan, "Detection of half broken rotor bar fault in VFD driven induction motor drive using motor square current MUSIC analysis", Mech. Syst. Signal Process., vol. 110, pp. 333-348, Sep. 2018.

[22] S. B. Lee et al., "Identification of False Rotor Fault Indications Produced by Online MCSA for Medium-Voltage Induction Machines", IEEE Transactions on Industry Applications, vol. 52, no. 1, pp. 729-739, Jan.-Feb. 2016

[23] A. K. Samanta, A. Naha, A. Routray and A. K. Deb, " Fast and accurate spectral estimation for online detection of partial broken bar in induction motors ", Mech. Syst. Signal Process., vol. 98, pp. 63-77, Jan. 2018.

[24] M. Drakaki, Y. L. Karnavas, A. D. Karlis, I. D. Chasiotis and P. Tzionas, "Study on fault diagnosis of broken rotor bars in squirrel cage induction motors: a multi-agent system approach using intelligent classifiers", IET Electric Power Applications, vol. 14, no. 2, pp. 245-255, Feb. 2020

[25] M. R. Mehrjou, N. Mariun, M. Hamiruce Marhaban, and N. Misron, "Rotor fault condition monitoring techniques for squirrel-cage induction machine-A review", Mech. Syst. Signal Process., vol. 25, no. 8, pp. $2827-$ 2848, Nov. 2011.

[26] W. T. Thomson and M. Fenger, "Current signature analysis to detect induction motor faults", IEEE Ind. Appl. Mag., vol. 7, no. 4, pp. 26-34, Jul. 2001

[27] K. N. Gyftakis, A. J. Marques Cardoso, and J. A. Antonino-Daviu, "Introducing the Filtered Park"s and Filtered Extended Park"s Vector Approach to detect broken rotor bars in induction motors independently from the rotor slots number", Mech. Syst. Signal Process., vol. 93, pp. 30-50, Sep. 2017.

[28] I. Antoniadou, G. Manson, W. J. Staszewski, T. Barszcz, and K. Worden, "A time-frequency analysis approach for condition monitoring of a wind turbine gearbox under varying load conditions", Mech. Syst. Signal Process., vol. 64-65, pp. 188-216, Dec. 2015.

[29] I. S. Bozchalooi and M. Liang, "Teager energy operator for multimodulation extraction and its application for gearbox fault detection", Smart Mater. Struct., vol. 19, no. 7, p. 075008, Jun. 2010.

[30] M. Liang and I. Soltani Bozchalooi, "An energy operator approach to joint application of amplitude and frequency-demodulations for bearing fault detection", Mech. Syst. Signal Process., vol. 24, no. 5, pp. 1473-1494, Jul. 2010. [31] Y. Imaouchen, M. Kedadouche, R. Alkama, and M. Thomas, "A Frequency-Weighted Energy Operator and complementary ensemble empirical mode decomposition for bearing fault detection", Mech. Syst. Signal Process., vol. 82, pp. 103-116, Jan. 2017.

[32] L. Noureddine, A. Hafaifa, and K. Abdellah, "Rotor fault detection of wind turbine squirrel cage induction generator using teager-kaiser energy operator", Control Intell. Syst., vol. 45, Mar. 2017.

[33] M. Pineda-Sanchez et al., "Application of the Teager-Kaiser Energy Operator to the Fault Diagnosis of Induction Motors", IEEE Trans. Energy Convers., vol. 28, no. 4, pp. 1036-1044, Dec. 2013.

[34] H. Teager, "Some observations on oral air flow during phonation", IEEE Trans. Acoust. Speech Signal Process., vol. 28, no. 5, pp. 599-601, Oct. 1980.

[35] J. F. Kaiser, "On a simple algorithm to calculate the "energy" of a signal", in International Conference on Acoustics, Speech, and Signal Processing, 1990, pp. 381-384 vol.1.

[36] P. Maragos, J. F. Kaiser, and T. F. Quatieri, "Energy separation in signal modulations with application to speech analysis", IEEE Trans. Signal Process., vol. 41, no. 10, pp. 3024-3051, Oct. 1993.

[37] R. B. Randall and W. A. Smith, "Uses and mis-uses of energy operators for machine diagnostics", Mech. Syst. Signal Process., vol. 133, p. 106199, Nov. 2019.

[38] R. B. Randall and W. Smith, "Application of the Teager Kaiser Energy Operator to Machine Diagnostics”, 2017.
[39] Li, T. Wang, Zhen, Gu, and Ball, "Modulation Sideband Separation Using the Teager-Kaiser Energy Operator for Rotor Fault Diagnostics of Induction Motors", Energies, vol. 12, p. 4437, Nov. 2019.

[40] A. Soualhi, G. Clerc, and H. Razik, "Detection and Diagnosis of Faults in Induction Motor Using an Improved Artificial Ant Clustering Technique", IEEE Trans. Ind. Electron., vol. 60, no. 9, pp. 4053-4062, Sep. 2013.

[41] J. Jung, J. Lee, and B. Kwon, "Online Diagnosis of Induction Motors Using MCSA”, IEEE Trans. Ind. Electron., vol. 53, no. 6, pp. 1842-1852, Dec. 2006.

[42] P. Mauri, "AC Induction Motor Slip", Plantservices.com. Retrieved 18 December 2012.

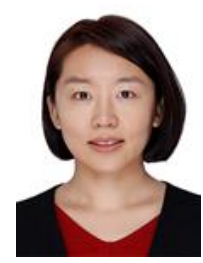

Haiyang Li received her B.S. and M.S. degree from Hebei University of Technology. She is currently a $\mathrm{PhD}$ student at the University of Huddersfield and sponsored by China Scholarship Council. Her research interests include fault diagnosis and condition monitoring for induction motors based on instantaneous electrical signatures.

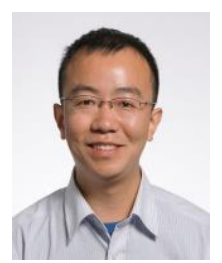

Guojin Feng received his B.S. and M.S. degree from Shandong University of Science and Technology, China in 2009 and 2012, respectively. In 2016, he obtained his Ph.D. degree in mechanical engineering at University of Huddersfield, UK. He is currently a Senior Research Fellow with the Centre for Efficiency and Performance Engineering (CEPE), University of Huddersfield. His research interests include intelligent wireless condition monitoring system for industrial rotating machines and on-rotor MEMS sensing systems.

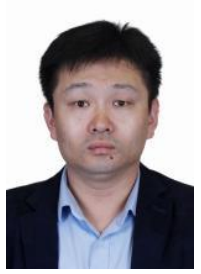

Dong Zhen received his B.Sc. degree in 2006 from Shandong University of Science and Technology, received his M.Sc. degree in 2009 from Shandong University of Science and Technology, received his Ph.D. degree in 2012 from University of Huddersfield, $\mathrm{He}$ is currently with the Hebei University of Technology, Tianjin, China. His research interests include advanced signal processing and rotating machine fault diagnosis.

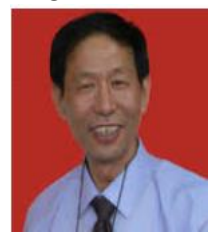

Fengshou Gu received the Ph.D. degree from the University of Manchester, Manchester, UK, in 2008. He is currently a Professor at the University of Huddersfield, Huddersfield, U.K. He is one of the experts in the fields of machinery diagnosis and vibroacoustic analysis, with over 30 years of research experience. His research interests include system modelling, various physical parameter measurements, advanced signal processing and fault diagnosis.

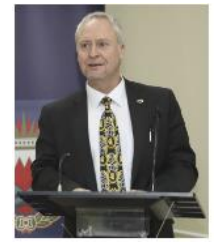

Andrew D. Ball obtained BEng degree from University of Leeds and received the $\mathrm{PhD}$ from the University of Manchester, UK. He is currently a Professor and Pro-ViceChancellor at the University of Huddersfield $(\mathrm{UoH})$. Also, he is the Director of the Centre of Efficiency and Performance Engineering (CEPE) at UoH. His research expertise is in the diagnosis of faults in mechanical, electrical and electro-hydraulic machines, and in data analysis and signal processing. 